\title{
Editorial: Introduction to the 38th Annual Gallery of Fluid Motion (Chicago, IL, USA 2020)
}

\author{
David H. Richter $\odot^{*}$ \\ Department of Civil and Environmental Engineering and Earth Sciences, University of Notre Dame, \\ Notre Dame, IN 46556, USA \\ Saverio E. Spagnolie ${ }^{\dagger}$ \\ Department of Mathematics, University of Wisconsin - Madison, Madison, WI 53706, USA
}

(Received 13 May 2021; published 15 November 2021)

DOI: 10.1103/PhysRevFluids.6.110001

The year 2020 was an abnormal year in many respects, and the 73rd Annual Meeting of the American Physical Society (APS) Division of Fluid Dynamics was no exception. Originally slated to be held in Chicago, IL from November 22-24, 2020, it was instead held entirely online due to the ongoing COVID-19 pandemic. Despite this interruption, including the necessary modifications to the overall conference program as it was shifted to a virtual format, the annual Gallery of Fluid Motion (GFM) was conducted as usual and suffered no shortage of compelling submissions. A number of submissions even spoke directly to the prominent challenge of COVID-19, bringing a fluid dynamics perspective to the task of better understanding and disrupting its spread.

The general timeline of GFM submissions was kept the same, requiring contributors to begin the submission process by September 16, 2020 to signal their intent, with final entries due by October 12, 2020. Ultimately, 60 video and 17 poster entries were submitted from 19 different countriesnumbers similar to previous, in-person meetings, speaking to the substantial robustness of the field as a whole. The virtual format's primary impact on the Gallery was naturally that there was no hall in which to display the poster and video entries. But the GFM website hosted by APS was set up as usual, where entries not only from 2020 but from previous years can still be viewed (https://gfm.aps.org).

As in past years, two panels were organized to judge the GFM entries, one for videos and one for posters. The video panel consisted of seven experts in the broad field of fluid mechanics and the poster panel consisted of three experts, and were led by the authors, respectively. Each entry was scored on a scale of 1-5. After a preliminary round of voting, an additional "finals" round was conducted to establish both the overall GFM winners as well as the Milton van Dyke Awards. The judging assignments were randomized and potential conflicts of interest were proactively avoided.

Below, we report the winners of the 2020 GFM competition (see also Fig. 1). The top three entries from each category were awarded the Milton van Dyke Award, which comes with a cash prize. Following this, the next three video entries were deemed GFM winners. Our congratulations to the winners! And we both congratulate and extend our gratitude to all the contributors for continuing in the strong tradition of making fluid mechanics informative, relevant, and visually stunning to a wide audience.

\footnotetext{
*david.richter.26@nd.edu

†spagnolie@math.wisc.edu
} 


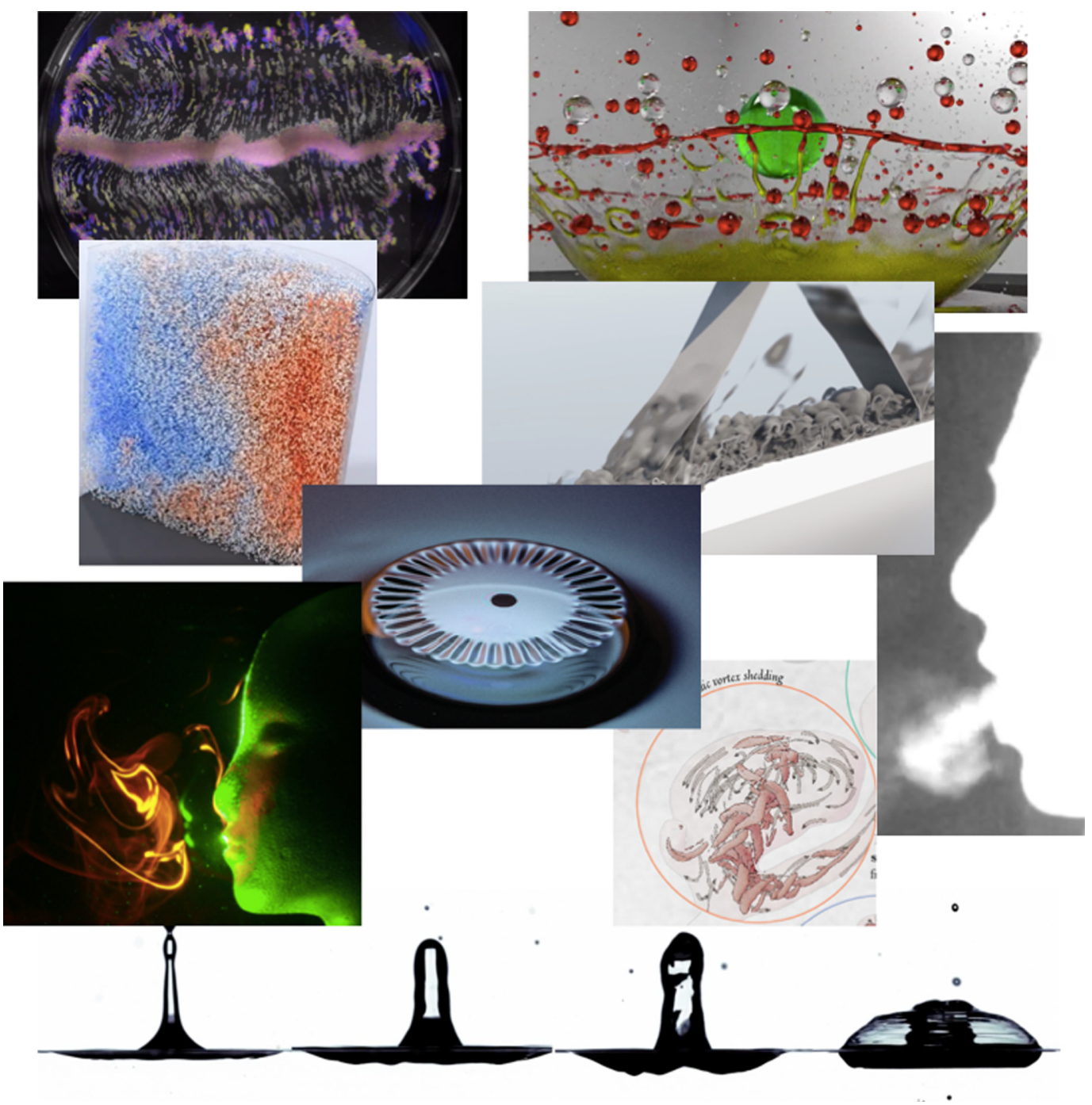

FIG. 1. Collage of the winning entries.

\section{Gallery of Fluid Motion Winners}

(1) Fuel Injection Supersonic Cavity

H. Sitaraman, N. Brunhart-Lupo, M. H. de Frahan, S. Yellapantula, B. Perry, J. Rood, R. Grout, M. Day, R. Binyahib, K. Gruchalla

National Renewable Energy Laboratory, USA; Lawrence Berkeley National Laboratory, USA

(2) Rayleigh-Taylor Instability in Drop Impact Experiments

$V$. Lherm, R. Deguen, T. Alboussière, M. Landeau

Université de Lyon, France; CNRS, Université Grenoble Alpes, France, Institut de Physique du Globe de Paris, France

(3) The Beauty of Turbulent Convection: A Particle Tracking Endeavor

P. Godbersen, J. Bosbach, D. Schanz, A. Schröder

Institute of Aerodynamics and Flow Technology, German Aerospace Center, Germany 


\section{Milton Van Dyke Award-Videos}

\section{(1) Rocket Yeast}

S. Atis, B. T. Weinstein, A. W. Murray, D. R. Nelson

Harvard University, USA; University of Chicago, USA

(2) Impact of High-Speed Diesel Drop Trains-Pursuing Cleaner Diesel Engines

D. Markt Jr., M. Raessi, A. Pathak, S.-Y. Lee, R. Torelli

University of Massachusetts Dartmouth, USA; Michigan Technological University, USA;

Argonne National Laboratory, USA

\section{(3) Air Flows in Opera}

P. Bourrianne, P. Kaneelil, M. Abkarian, H. A. Stone

Princeton University, USA; CNRS, Université de Montpellier, France

\section{Milton Van Dyke Award-Posters}

(1) Spectral Landscapes of Flow Instabilities in Brain Aneurysms

T. Natarajan, D. MacDonald, L. Temor, P. Coppin, D. Steinman

University of Toronto, Canada; Ontario College of Art and Design, Canada

(2) Viscous Wrinkling of Non-uniform Sheets

O. McRae, A. Oratis, J. Bird

Boston University, USA

\section{(3) Fluid Dynamics of COVID-19 Spread}

C. Kovar, L. Panczner, H. Reuter, A. Eslam-Panah

Pennsylvania State University, USA

Finally, we thank the local organizing committee, particularly Jonathan Freund and Randy Ewoldt, for their immense efforts in transitioning the conference to a virtual format and for helping address the specific needs of the GFM. We also thank Ken Kiger for his continued support of the GFM, providing indispensable advice and institutional knowledge. We also thank APS, especially Paul Dlug, for overseeing the GFM submission and host sites, as well as Monica Malouf at Meetings and More for help in organizing the GFM website. 